\title{
Comparison of the Effects of Velocity and Range Triggers on Trajectory Dispersions for the Mars 2020 Mission
}

\author{
Soumyo Dutta* and David W. Way* \\ NASA Langley Research Center, Hampton, VA 23681, USA
}

\begin{abstract}
Mars 2020, the next planned U.S. rover mission to land on Mars, is based on the design of the successful 2012 Mars Science Laboratory (MSL) mission. Mars 2020 retains most of the entry, descent, and landing (EDL) sequences of MSL, including the closed-loop entry guidance scheme based on the Apollo guidance algorithm. However, unlike MSL, Mars 2020 will trigger the parachute deployment and descent sequence on range trigger rather than the previously used velocity trigger. This difference will greatly reduce the landing ellipse sizes. Additionally, the relative contribution of each models to the total ellipse sizes have changed greatly due to the switch to range trigger. This paper considers the effect on trajectory dispersions due to changing the trigger schemes and the contributions of these various models to trajectory and EDL performance.
\end{abstract}

\section{Introduction}

The Mars 2020 mission is a proposed rover mission that will land on the red planet in February 2021. ${ }^{1}$ The Mars 2020 entry, descent, and landing (EDL) architecture is heavily based on the successful Mars Science Laboratory (MSL) mission, which accomplished several firsts in Mars exploration history, such as landing the largest payload $(900 \mathrm{~kg})$, deploying the largest supersonic parachute (21.5 m diameter), and having the smallest landing footprint (less than $10 \mathrm{~km}$ radius) for Mars EDL mission. ${ }^{2,3}$ The Mars 2020 mission to continue the in situ science conducted by MSL, but also adds goals to acquire a diverse set of samples and cache them for potential return to Earth in the future. All of these sciences goals will be achieved by leveraging the MSL mission architecture while improving landing technology to allow closer proximity delivery to scientifically important sites. ${ }^{1}$ Fig. 1 shows the MSL EDL concept of operations which is very similar to the current Mars 2020 concept of operations.

As mentioned earlier, an achievement of the MSL mission was a small predicted landing footprint around the targeted location when compared to past Mars lander missions, such as the 2008 Phoenix lander (which had an ellipse footprint of $55 \mathrm{~km}$ radius). ${ }^{5}$ MSL was able to achieve such a small landing footprint by using a closed-loop entry guidance during the hypersonic and supersonic phases of flight and deploying the parachute based on this guidance scheme. ${ }^{6}$ The guidance scheme utilized for MSL is based on Apollo guidance flown during lunar return missions and the reader is referred to Refs. 6-7 for a more detailed explanation of the scheme. The final parachute triggering scheme used for MSL was based on a velocity trigger, which meant that the parachute was deployed at a chosen planet-relative velocity corresponding to a desired parachute opening Mach number. Targeting a planet-relative velocity for parachute deployment leads to usually very tight tolerance on the actual deployed velocity, but it also leads to a corresponding increase in dispersion of the range from the landing target (position) at which the parachute is deployed.

This dispersion in range from target is caused by the many combinations of position, attitude, and flight path angle that can lead an EDL trajectory to the same planet-relative velocity. After parachute deploy, the dispersion in range to target usually grows while the vehicle is on the parachute, since the vehicle range is not controlled during this phase of the flight and winds tend to blow the vehicle in downrange and crossrange directions. Hence, although MSL's used of a closed-loop entry guidance scheme increased landing footprint

*Aerospace Engineer, Atmospheric Flight and Entry Systems Branch, 1 N. Dryden St., and AIAA Member. 


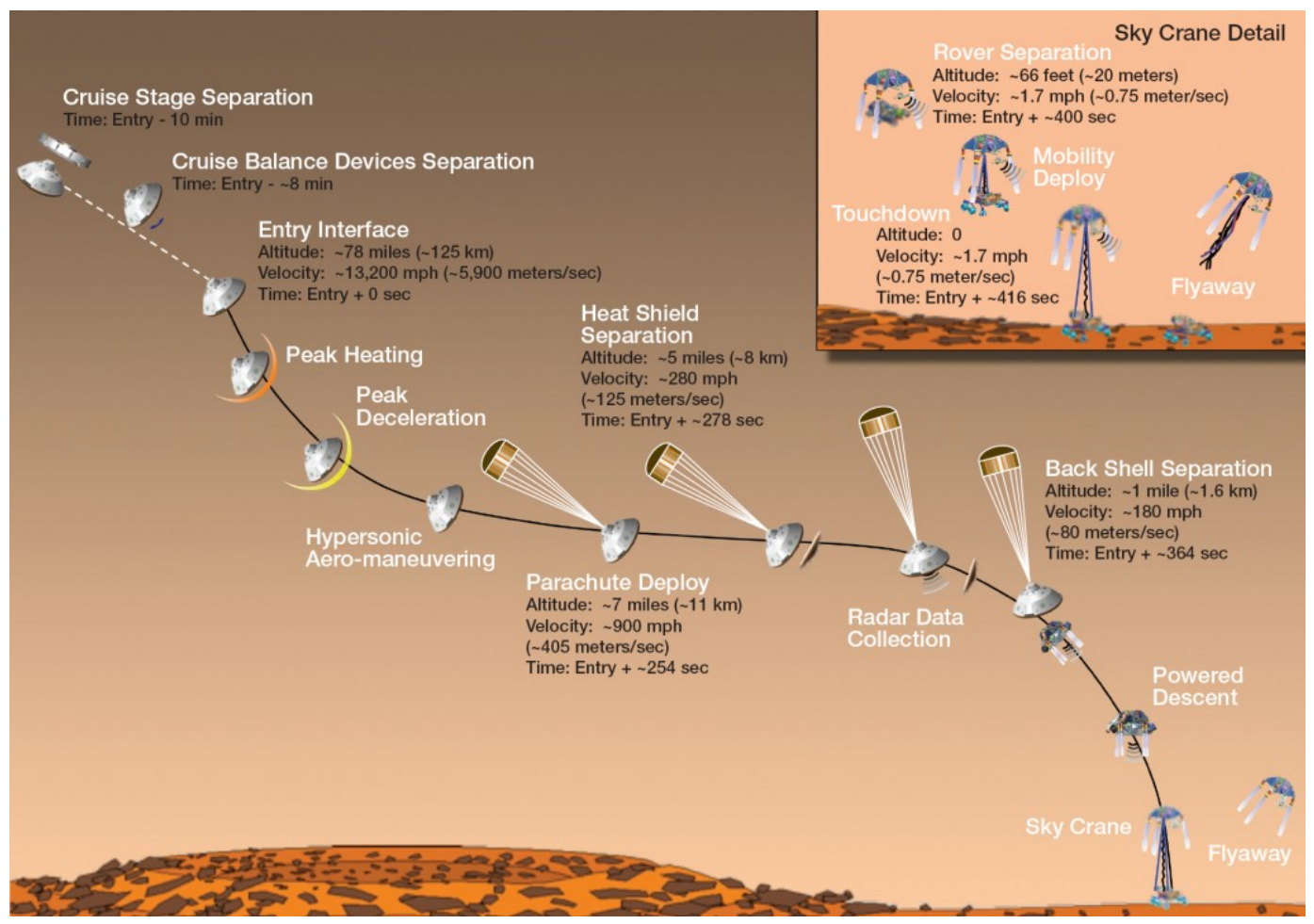

Figure 1. Mars Science Laboratory entry, descent, and landing sequence. ${ }^{4}$ Mars 2020 concept of operations will be very similar to the MSL sequence.

accuracy when compared to previous uncontrolled Mars hypersonic entry sequences, the residual range error at parachute deploy caused by the use of a velocity trigger grew during the parachute phase of the flight.

The range error of the velocity trigger could be further reduced if the parachute deployment trigger is triggered based on a range from a target instead of planet-relative velocity. This range can be correlated to a desired peak opening Mach number, thus still achieving desired parachute deploy conditions. Additionally, the range trigger provides a tighter tolerance on the range at which the parachute is deployed when compared to the velocity trigger, leading to a very small footprint in range space at parachute deploy. Thus, although range errors grow during the uncontrolled time on parachute phase, the final landing footprint for the range trigger is smaller than the velocity trigger at the same trajectory conditions. Intuitively, one would expect range triggers to have a larger dispersion on parachute deploy velocity, but past work has shown correlations between wind speed and planet-relative velocity at parachute deploy mitigates the expected increase in the dispersion of the parachute deploy Mach number. ${ }^{8}$

Range trigger was considered as an option for the parachute triggering mechanism for MSL during initial design, but ultimately due to some perceived risk considerations, velocity trigger was selected. ${ }^{8}$ However, the Mars 2020 mission has already selected range trigger as its parachute deploy triggering scheme. The change in parachute deploy triggering scheme itself leads to landing ellipses of the size of 7-9 km radius and potentially even smaller ellipses at certain landing sites. These numbers can be compared to $12-16 \mathrm{~km}$ major axis size of velocity trigger-based landing ellipses for MSL. Correspondingly, the reduction in ellipse size and the change in parachute deploy triggering logic leads to changes in the relative contribution of various models to the total trajectory dispersions and their effect on the final footprint sizes. This paper considers the sensitivity of these contributing models and their effects on trajectory dispersions.

\section{Simulation Detail}

The Program to Optimize Simulated Trajectories II (POST2) is the tool used for end-to-end EDL simulations for the Mars 2020 mission. POST2 is a six degree-of-freedom flight dynamics simulation tool that can simultaneously simulate the trajectory of up to 20 independent or connected rigid bodies. It is a generalized 
point mass, discrete-parameter targeting and optimization trajectory simulation program with multi-vehicle capabilities that integrates translational and rotational equations of motion along the trajectory. The simulation tool has significant EDL flight heritage as it has been used in the past successfully for several Mars EDL missions, such as Mars Pathfinder, ${ }^{9}$ Mars Exploration Rovers, ${ }^{10}$ Mars Phoenix, ${ }^{11}$ and Mars Science Laboratory. ${ }^{12}$

The Mars 2020 simulation is a continuation of the POST2-based MSL end-to-end EDL simulation developed at the NASA Langley Research Center. The simulation starts approximately $50 \mathrm{~s}$ after Cruise Stage Separation and models the trajectories of fourteen independent bodies, such as the descent stage, parachute, backshell, heatshield, rover, and even the ejected balance masses. Interaction between interconnected bodies, such as the parachute and the aeroshell or the descent stage and the rover are modeled using multi-body force models originally developed for the Mars Exploration Rovers' simulations. ${ }^{10,12}$

The end-to-end simulation, which incorporates vehicle, planet, and atmospheric models, is used during mission planning to assess the system's performance against requirements and response to off-nominal conditions. In order to quantify the robustness of the system, Monte Carlo analysis is conducted using the simulation. A pre-set group of input variables are stochastically dispersed and statistics on metrics of interest are tracked at specific EDL events. The simulation uses site specific atmospheric parameters - density, temperature, pressure, and horizontal winds - generated by mesoscale atmospheric models. ${ }^{13}$ The use of a physics-based atmospheric profiles, such as mesoscale based data, in the simulation was novel to MSL and the Mars 2020 simulation continues that usage. The output metrics are collected for multiple runs of the simulation.

In this paper, this end-to-end simulation is exercised to compare the Mars 2020 EDL trajectory using the two different triggers. Specifically, the effect of the various input dispersion models on the footprint ellipses is considered.

\section{Ellipse Contributions}

\section{A. Velocity Trigger}

The Mars 2020 POST2 EDL simulation has 800 input variables that are stochastically dispersed during Monte Carlo analysis. These input variables consist of a variety of sources like initial state dispersions, atmospheric variations, uncertainties in aerodynamics and mass properties, gravitational acceleration dispersions, and navigation and controls errors. However, not all of these input sources are equal contributors to trajectory dispersions, especially considering the trajectory envelope at various EDL phases. Previous work has identified some of the top contributors to be aerodynamic uncertainties, atmospheric variations, and initial state errors. However, due to the Mars 2020 guidance, navigation, and control (GN\&C) scheme, the trajectory footprints are also sensitive to the attitude initialization error prior to cruise stage separation. The attitude initialization error is a knowledge error in the attitude of the vehicle from the start of operations of the EDL GN\&C, and the integrated altitude rate that is used by the entry guidance scheme for range flown ${ }^{3}$ is very sensitive to the initial attitude error.

Figures 2-3 show the contribution of various models on the trajectory footprint in downrange and crossrange directions at various events during EDL when velocity trigger is used. A separate Monte Carlo analysis was conducted one at a time with dispersions from only a single model to determine that model's individual contribution to the trajectory envelope. The Monte Carlo dispersions considered for each model have been presented in past works, such as Refs. 12 and 14. The results in Figs. 2-3 were generated using simulation settings for Gale crater, ${ }^{12}$ which was the landing location of MSL. Various EDL events are marked using vertical lines in figures. These events are entry interface (EI), start of range control (RC), start of first bank reversal (Rev1), end of the first bank reversal (End1), start of heading alignment (HDA), start of the parachute trigger (SUFR), actual parachute deployment (PD), heatshield separation (HS), backshell separation (BS), and touchdown (TD). The actual timeline for these events would be different for each case on the Monte Carlo analysis, but for simplicity the timeline of the nominal case is used to display the results. Finally, the footprint size variances $\left(\sigma^{2}\right)$ shown in downrange and crossrange directions are calculated using Choselky decomposition of the covariance matrix. The percentage contribution is based on a root sum of squares of the various variances stacked together.

Upon comparing the variance values in Figs. 2 and 3, one sees that ellipse sizes are largest in the downrange direction at most EDL events for the velocity trigger. This is expected as downrange is the dominant direction of travel during EDL and hence errors are expected to propagate more in that axis. 


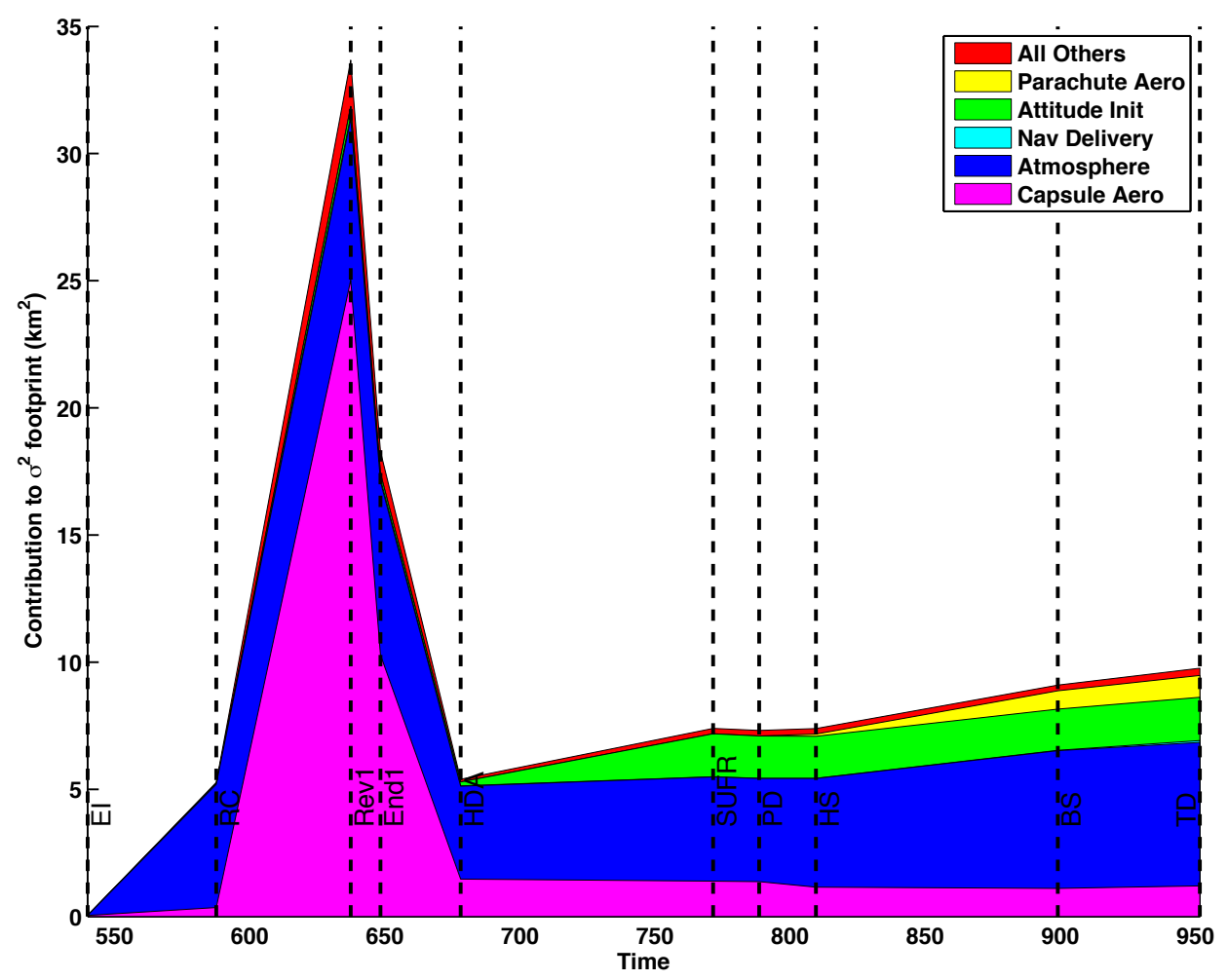

(a) Variance

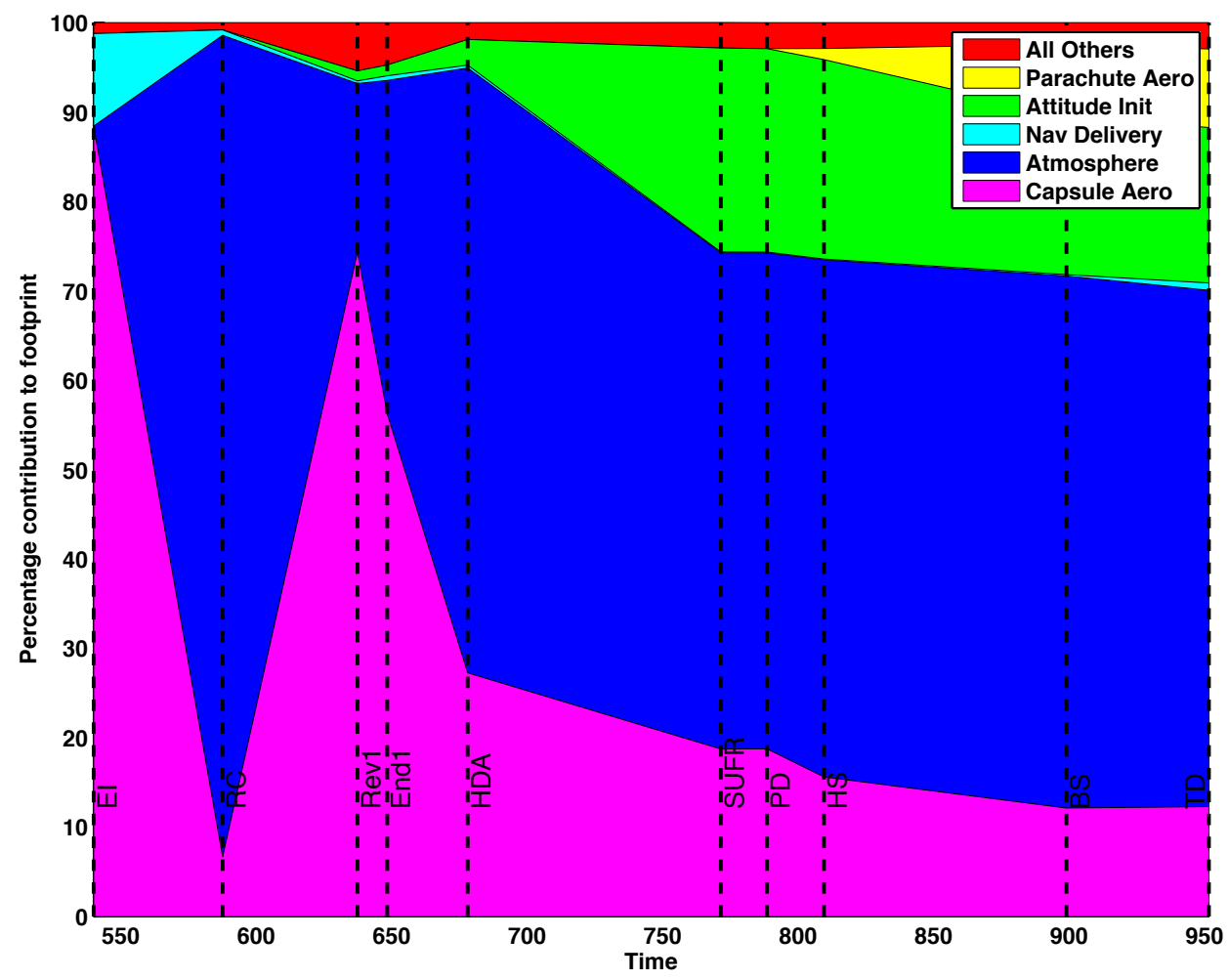

(b) Percentage

Figure 2. Contributions in the downrange direction to the ellipse size when using velocity trigger. 


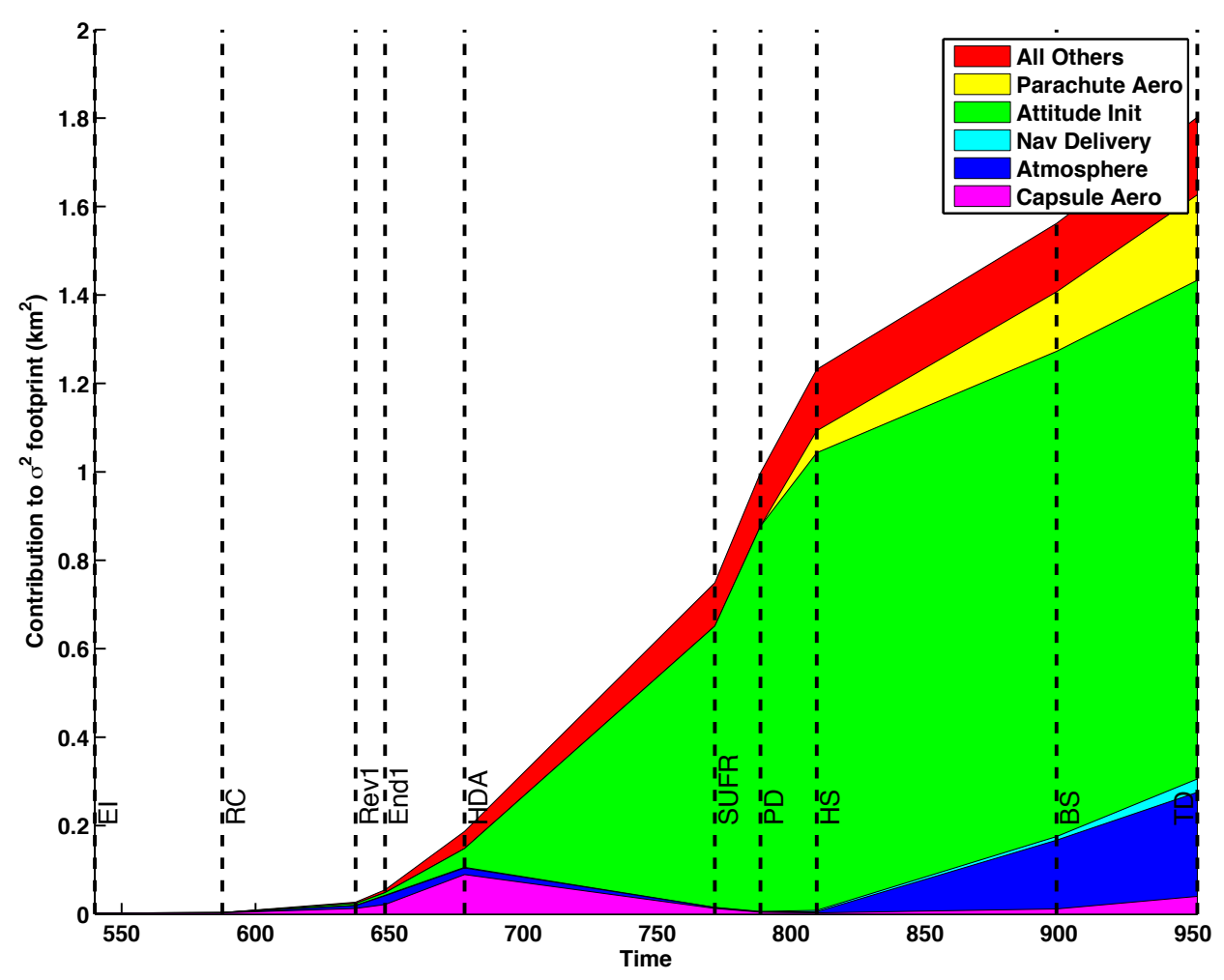

(a) Variance

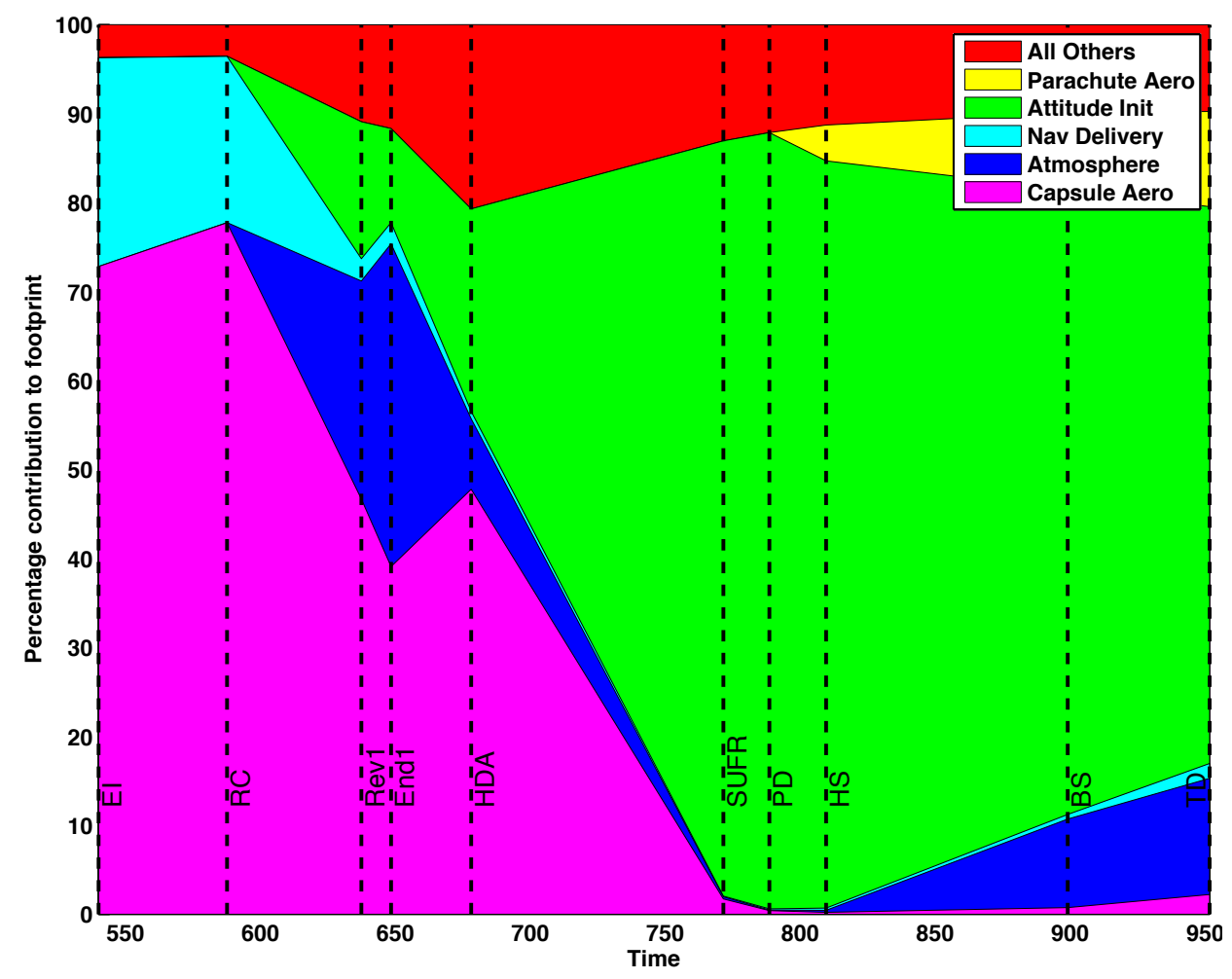

(b) Percentage

Figure 3. Contributions in the crossrange direction to the ellipse size when using velocity trigger. 
Additionally, one can see the advantage of the closed-loop entry guidance as errors propagated during range control (RC) are reduced by the bank reversals (Rev1 and End1), leading to a smaller error at the start of heading alignment (and the end of range control). If this was uncontrolled guided entry, the errors in the capsule aerodynamics and atmosphere would continue to increase throughout the entry phase. This underscores the rationale behind the large improvement MSL achieved over the preceding ballistic, Mars EDL missions in landing accuracy.

The largest contributor to footprint size, in general, are atmospheric dispersions, with wind dispersions being larger contributors than density. During the range control phase, uncertainties in the capsule aerodynamics trump atmospheric uncertainties, but due to the closed-loop control these errors are flown out. After parachute trigger (Stand-up-and-fly-right or SUFR) and parachute deployment (PD), capsule aerodynamics has a very small effect $\left(<2 \mathrm{~km}^{2}\right)$ on the overall footprint dispersions. Between parachute deployment and backshell separation (BS), which is the phase during which the vehicle is on the parachute, one sees that parachute aerodynamics is a small contributor to overall footprint size, especially in the crossrange direction. However, atmospheric dispersions still serve as the largest contributors, accounting for approximately $70 \%$ of the footprint at touchdown.

The large contributions of aerodynamic and atmospheric uncertainties to footprint sizes are expected. What may be unexpected is the lack of larger contributions of the initial state error (Nav Delivery) in the downrange direction. MSL had very accurate initial states when compared to other Mars missions, which was aided by the orbital determination during cruise and prior to entry that narrowed down the initial state. ${ }^{15}$ Hence, due to the small value of these dispersions, they have very small effect on downrange variation.

Finally, another unexpected contributor to the footprint dispersion is the attitude initialization error. As explained earlier, this is knowledge error in the vehicle GN\&C about its own attitude, and the integrated effect is especially seen in the crossrange direction. Since the primary motion of the vehicle is in the downrange direction, attitude error leads to dispersion in the perpendicular direction to travel - crossrange axis.

\section{B. Range Trigger}

Figures 2 and 3 give a sense of the contributions to ellipse sizes for the MSL as-flown velocity triggering scheme. Figures 4 and 6 show the ellipse sizes at various EDL events when the range trigger is used to deploy the parachute. This architecture gives a sense of predicted vehicle performance for the Mars 2020 mission. Figure 5 shows a zoomed-in view of Fig. 4(a) showing the variance of the ellipse size in the downrange direction. Similar to velocity trigger results, the simulation settings used for the range trigger results are tuned for Gale crater for ease of comparison.

Similar to the situation for the velocity trigger, the downrange axis has larger dispersions at most of the EDL events compared to the crossrange axis. However, one important difference is the shape of the downrange ellipse size at parachute trigger point (SUFR). At this location, as seen in the zoomed view presented in Fig. 5, the error is extremely small $\left(<0.25 \mathrm{~km}^{2}\right)$ especially when compared to the ellipse size at the same trajectory event (about $7 \mathrm{~km}^{2}$ ) using velocity trigger as was shown in Fig. 2. This is of course the advantage of the range trigger scheme, since the parachute deploy point is selected based on a rangeto-go to the target and this leads to small dispersions in the range at parachute deploy trigger point. The Apollo guidance scheme utilized by MSL and Mars 2020 uses the downrange direction as the range value for the trigger. ${ }^{6,7}$ Thus, the guidance limits the error in the downrange direction, but there is still some small, residual dispersions in the crossrange direction at the parachute trigger point despite the reduction in dispersion in the downrange direction. This leads to an inversion in traditional thinking of ellipse orientation at the parachute trigger point, since with the range trigger the footprint is larger along the crossrange direction than the downrange direction at the the trigger point.

Another interesting observation is the similarity of the crossrange ellipse distributions for range trigger to the velocity trigger counterparts. Figs. 3 and 6 are really indistinguishable from each other. The similarity between the two is because the range trigger for Apollo guidance is based on values in the downrange direction, so there is very little effect in the crossrange dispersion. This underscores some potential avenues for improvement for future hypersonic entry guidance schemes that can achieve the accuracy in the downrange axis like range trigger-based Apollo guidance but also mitigate dispersions in the crossrange direction.

As was seen in the velocity trigger case, atmospheric dispersions play a large contribution to the ellipse size, both in downrange and crossrange directions. However, the lack of a large contribution of the capsule aerodynamics to the landed footprint is an interesting observation from Figs. 4-6. In the past, aerodynamic uncertainties of the aeroshell during the hypersonic and supersonic phases of the flight had a large effect on 


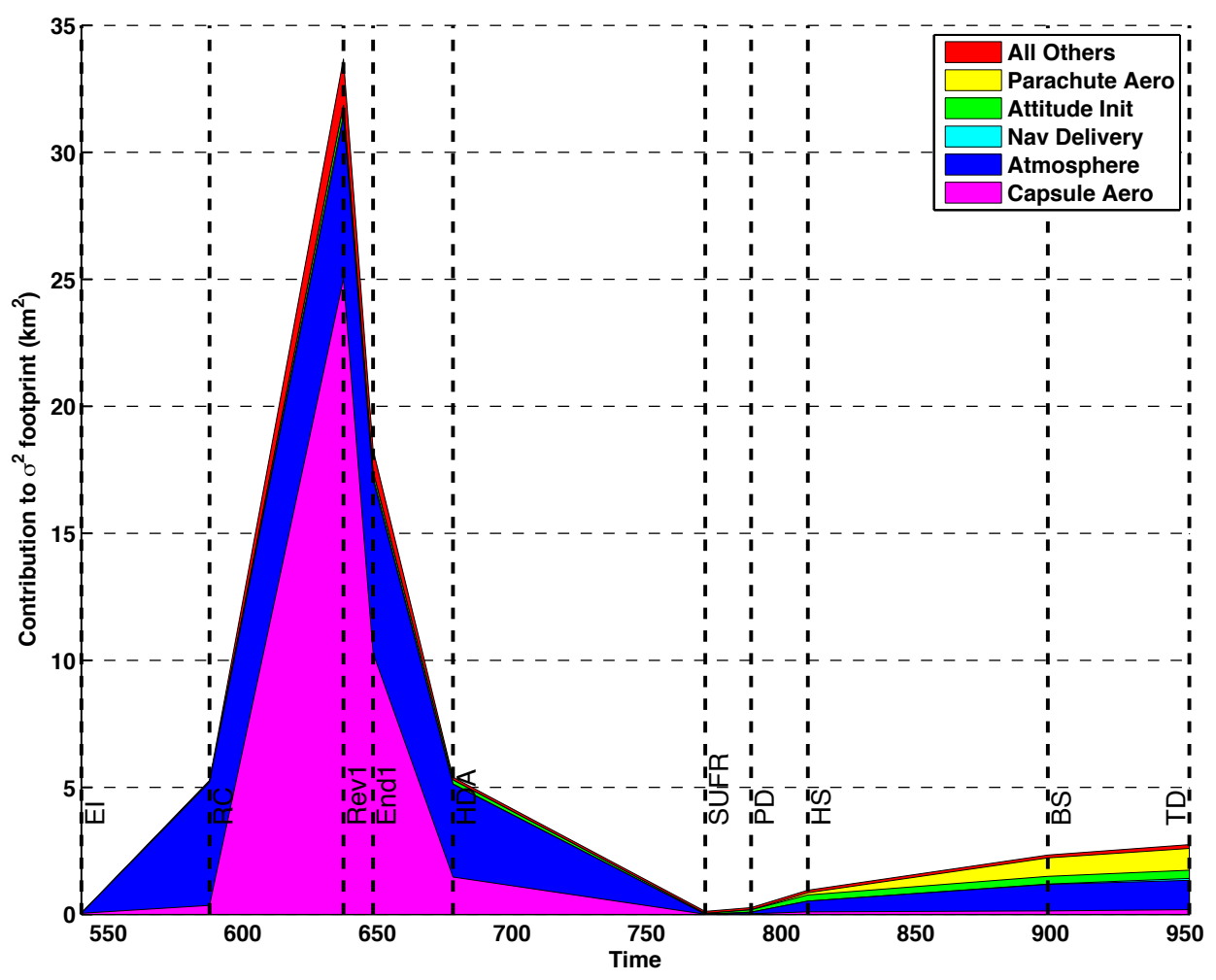

(a) Variance

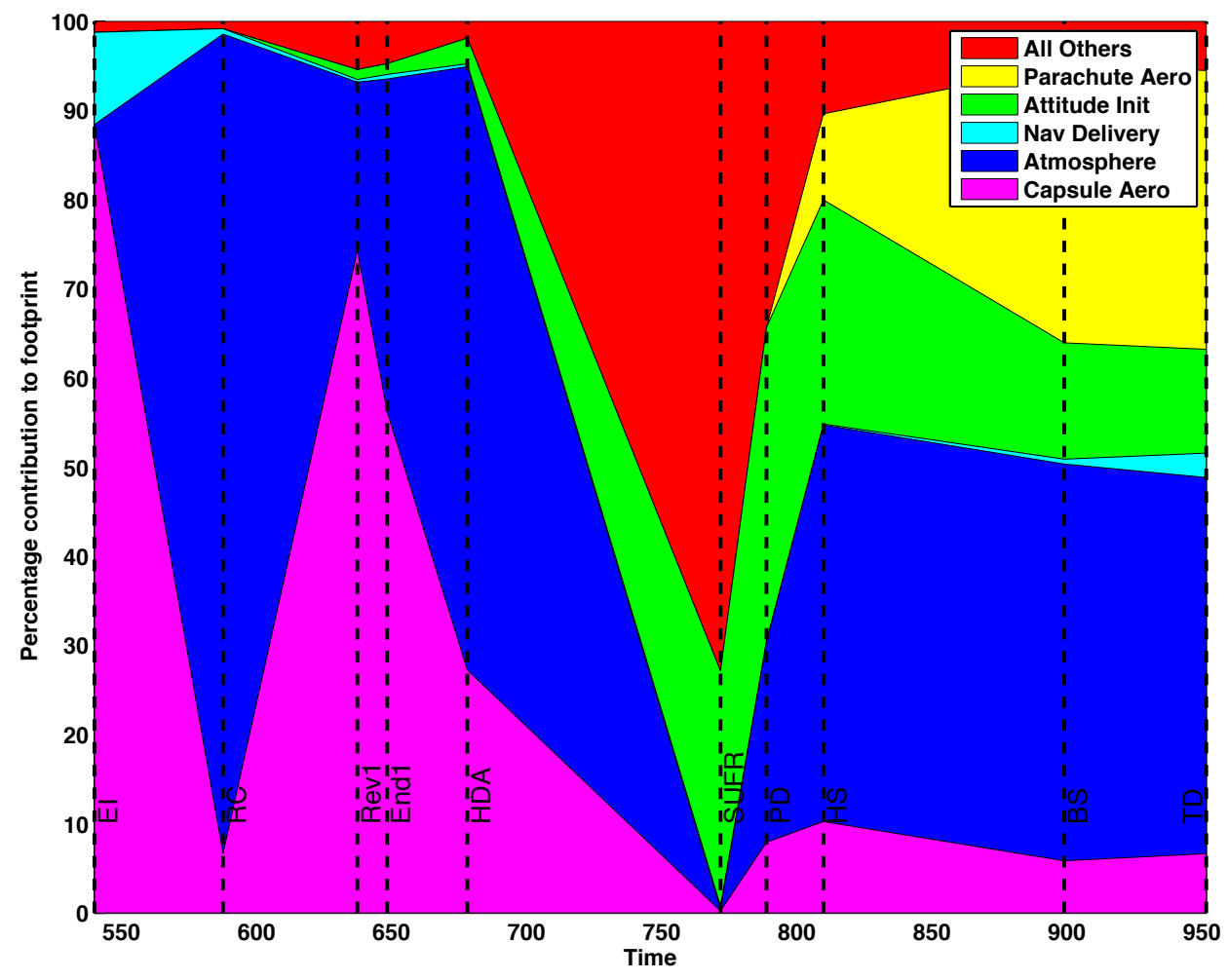

(b) Percentage

Figure 4. Contributions in the downrange direction to the ellipse size when using range trigger. 


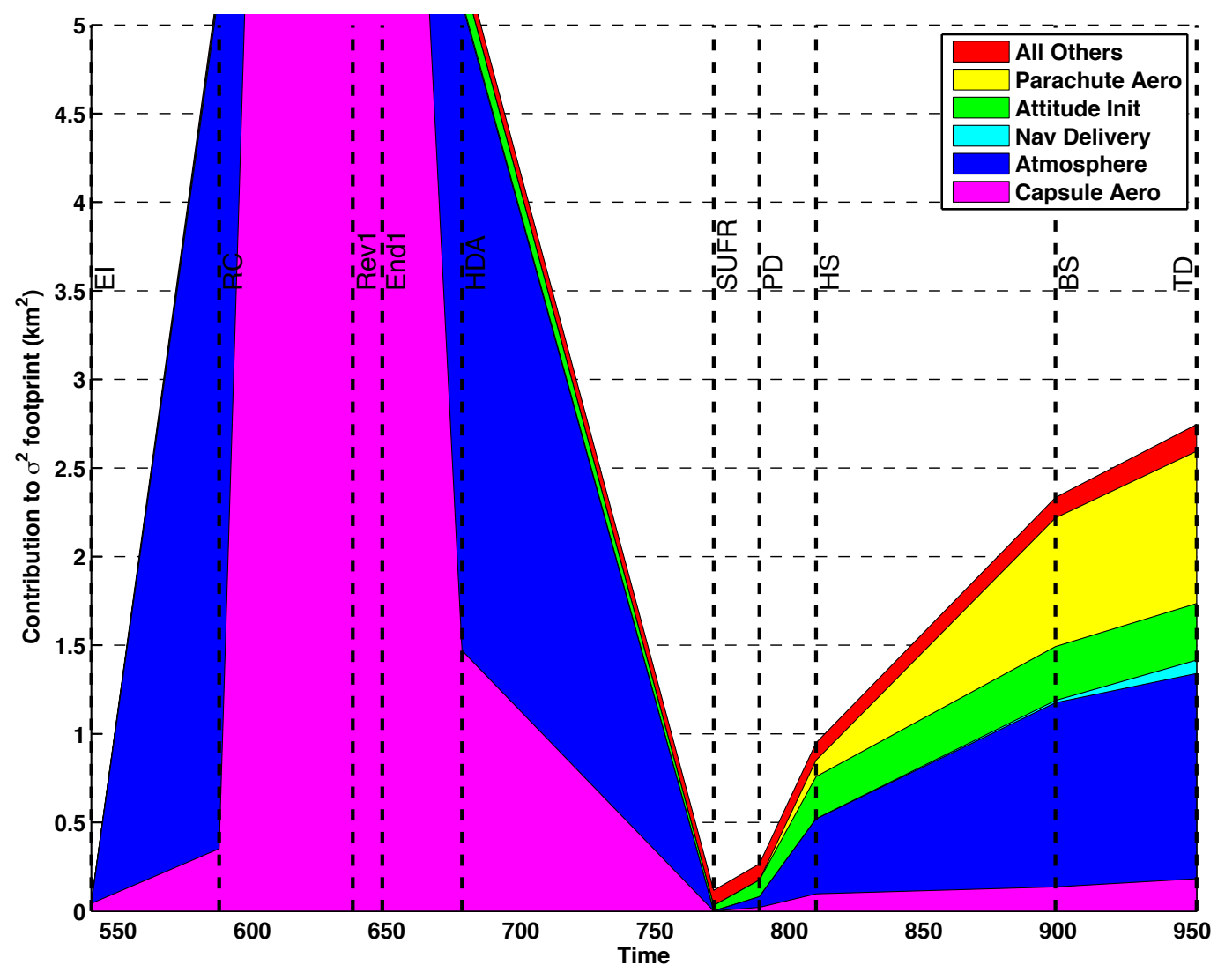

Figure 5. Zoomed-in view of the contributions in the downrange direction to the variance of the ellipse size.

the landed footprint size and trajectory design considerations. In fact for ballistic entries and uncontrolled lifting bodies, the aeroshell aerodynamics is one of the largest contributors to footprints. ${ }^{6,7}$ However, the type of interplanetary trajectory knowledge expected for more recent Mars missions, such as vastly improved delivery states, ${ }^{15}$ and the use of range triggers, diminishes the downstream effect of hypersonic and supersonic aeroshell aerodynamics. One still sees a small contribution of the capsule aerodynamics on the footprint size in Figs. 2-5 growing after parachute deployment (PD), but these are simply due to some small integrative effects of attitude error caused by aerodynamic uncertainties.

\section{Implications}

The ellipse contributions as variances of several modeling parameters at the parachute trigger event and touchdown are summarized in Tables 1 and 2. These tables show the effect of these parameters on the trajectory envelope at two critical events during EDL and the percentage difference in variances going from velocity trigger to the range trigger. Looking at Table 1 , one can see a large reduction in the downrange error size when range trigger is used. Incidentally, the Apollo Guidance scheme with range trigger does not lead to any appreciable difference in the ellipse size in the crossrange direction.

The effectiveness of the range trigger is also visible in the statistics of the ellipse size at touchdown. For most modeling parameters, there is a decrease in ellipse size when using the range trigger rather than the velocity trigger. However, when one compares the range trigger downrange variances between the parachute trigger event and touchdown, one can see a large increase for several modeling parameters. The largest percentage increase is for the atmospheric modeling parameter, whose ellipse contribution variance increases from $0.0003 \mathrm{~km}^{2}$ at parachute deploy to $1.16 \mathrm{~km}^{2}$ at touchdown. This underscores an important lesson from this exercise. For an EDL architecture reliant on parachutes, atmospheric uncertainties that can be mitigated during the entry phase by a well-tuned entry guidance scheme will still be a large contributor to the ellipse size at touchdown due to the increase in downrange and crossrange errors during the uncontrolled 


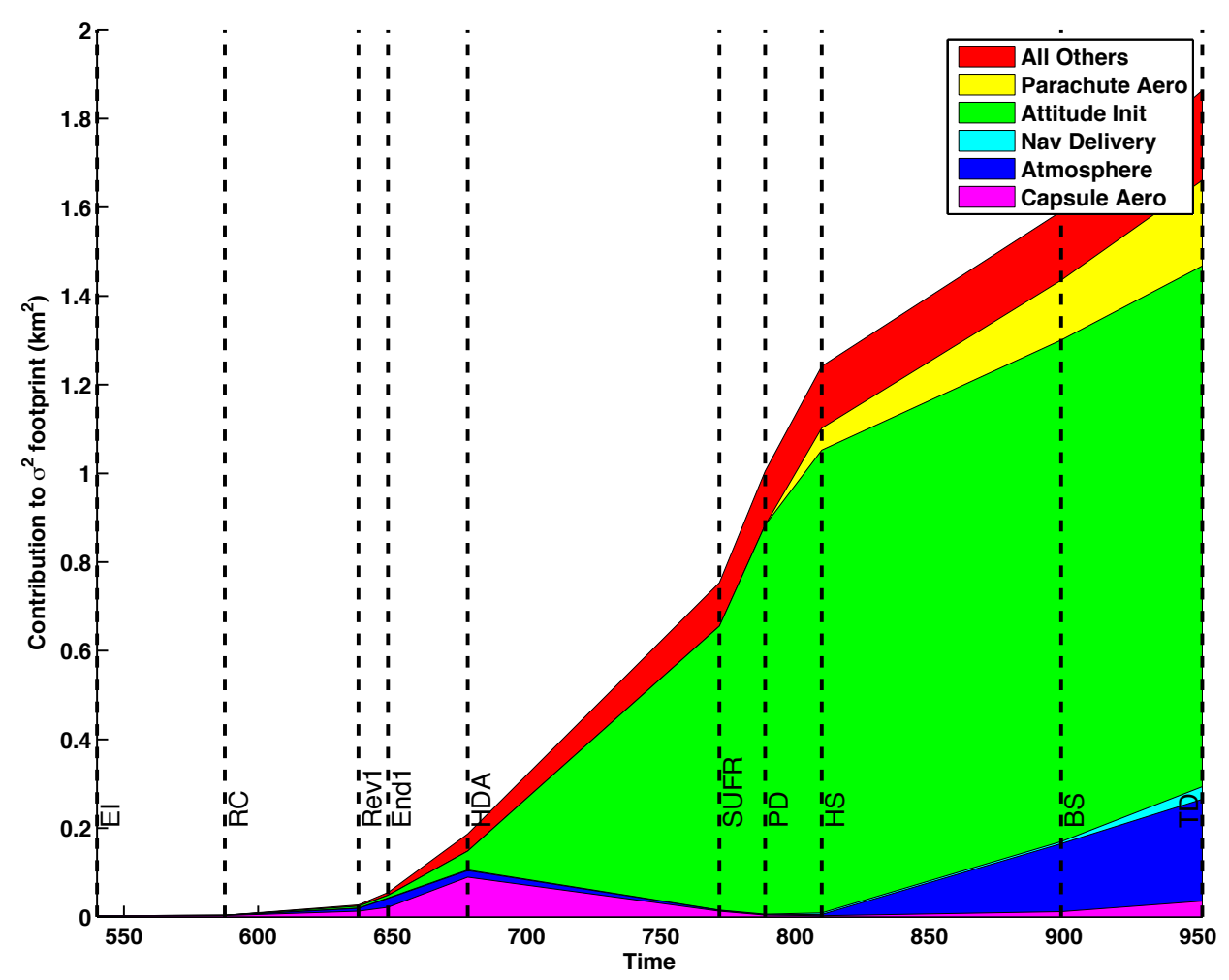

(a) Variance

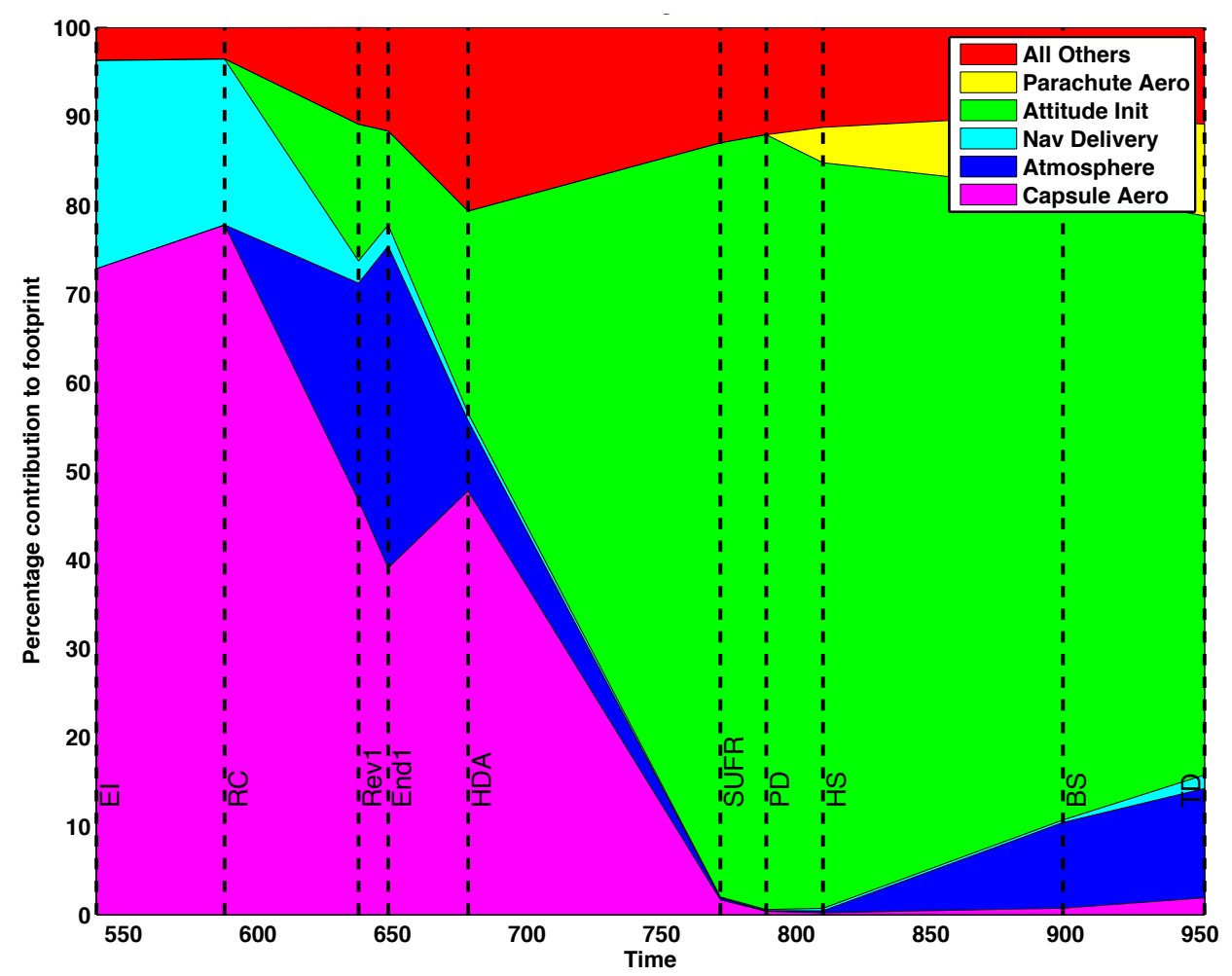

(b) Percentage

Figure 6. Contributions in the crossrange direction to ellipse size when using range trigger. 
Table 1. Contribution to ellipse size at the parachute trigger event from velocity trigger and range trigger.

\begin{tabular}{l|c|c|c|c|c|c}
\hline \hline Modeling Parameter & $\begin{array}{c}\text { Vel. Trigger } \\
\text { Downrange } \\
\text { Variance } \\
\left(\mathrm{km}^{2}\right)\end{array}$ & $\begin{array}{c}\text { Vel. Trigger } \\
\text { Crossrange } \\
\text { Variance } \\
\left(\mathrm{km}^{2}\right)\end{array}$ & $\begin{array}{c}\text { Range Trigger } \\
\text { Downrange } \\
\text { Variance } \\
\left(\mathrm{km}^{2}\right)\end{array}$ & $\begin{array}{c}\text { Range Trigger } \\
\text { Crossrange } \\
\text { Variance } \\
\left(\mathrm{km}^{2}\right)\end{array}$ & $\begin{array}{c}\text { \% Diff. in } \\
\text { Downrange }\end{array}$ & $\begin{array}{c}\% \text { Diff in } \\
\text { Crossrange }\end{array}$ \\
\hline Atmosphere (Wind and Density) & 4.1050 & 0.0019 & 0.0003 & 0.0019 & -99.99 & -0.53 \\
Capsule Aerodynamics & 1.3850 & 0.0128 & 0.0003 & 0.0125 & -99.98 & -2.04 \\
Attitude Initialization & 1.6850 & 0.6362 & 0.0307 & 0.6406 & -98.18 & 0.69 \\
Navigation Delivery Error & 0.0070 & 0.0005 & 0.0004 & 0.0006 & -94.57 & 13.46 \\
All Other Dispersions & 0.2120 & 0.0978 & 0.0848 & 0.0980 & -60.00 & 0.20 \\
\hline \hline
\end{tabular}

Table 2. Contribution to ellipse size at touchdown from velocity trigger and range trigger.

\begin{tabular}{l|c|c|c|c|c|c}
\hline \hline Modeling Parameter & $\begin{array}{c}\text { Vel. Trigger } \\
\text { Downrange } \\
\text { Variance } \\
\left(\mathrm{km}^{2}\right)\end{array}$ & $\begin{array}{c}\text { Vel. Trigger } \\
\text { Crossrange } \\
\text { Variance } \\
\left(\mathrm{km}^{2}\right)\end{array}$ & $\begin{array}{c}\text { Range Trigger } \\
\text { Downrange } \\
\text { Variance } \\
\left(\mathrm{km}^{2}\right)\end{array}$ & $\begin{array}{c}\text { Range Trigger } \\
\text { Crossrange } \\
\text { Variance } \\
\left(\mathrm{km}^{2}\right)\end{array}$ & $\begin{array}{c}\text { \% Diff. in } \\
\text { Downrange } \\
\text { Variance }\end{array}$ & $\begin{array}{c}\% \text { Diff in } \\
\text { Crossrange } \\
\text { Variance }\end{array}$ \\
\hline Atmosphere (Wind and Density) & 5.64 & 0.24 & 1.16 & 0.23 & -79.46 & -2.41 \\
Capsule Aerodynamics & 1.20 & 0.04 & 0.18 & 0.04 & -84.81 & -11.62 \\
Parachute Aerodynamics & 0.86 & 0.19 & 0.86 & 0.19 & 0.00 & 0.00 \\
Attitude Initialization & 1.70 & 1.13 & 1.17 & 0.32 & -30.90 & -71.63 \\
Navigation Delivery Error & 0.08 & 0.03 & 0.08 & 0.03 & -5.06 & -5.65 \\
All Other Dispersions & 0.29 & 0.18 & 0.20 & 0.15 & -29.86 & -14.03 \\
\hline \hline
\end{tabular}

parachute phase of flight.

The lack of sensitivity of the footprint size to capsule aerodynamics has a consequence for the design of future entry guidance schemes as well. In general, entry guidance schemes are designed to fly out aerodynamic errors and atmospheric uncertainty-caused errors in the drag profile. With a more precise control over the actual drag profile flown, the entry guidance reduces dispersions in range and position at the terminal control point - for example the parachute deploy point. This leads to small dispersion of the footprint at the guidance terminal control point and correspondingly at touchdown. However, with range triggers and modern Mars EDL entry delivery dispersions, the dispersions at entry terminal control point are already very small, so the landing dispersions are unaffected by capsule aerodynamic uncertainties. Improving entry guidance schemes will not show any appreciable improvement in landing footprint size, especially for systems that have uncontrolled terminal phase of flight such as parachute descent. As one strives for pinpoint accuracy for future Mars missions, such sub-100 m ellipses, the uncertainties to tackle will be atmospheric-related errors that propagate once the parachute or terminal phase has been initiated. These results suggest that range control during parachute flight or improved control authority during terminal descent and landing are the best ways to further reduce landing footprint sizes.

Another consequence of the range trigger and the reduction in the landing ellipse size has been a change to the approach of landing site selection. Since the landing footprint can smaller by a factor of two between the velocity trigger based architecture used for MSL and the range trigger based architecture being employed by Mars 2020, several landing sites that could not accommodate a MSL landing ellipse are being considered as potential Mars 2020 landing sites. In many cases for Mars 2020, the landing ellipses can be placed very close to the scientific areas of interest which was not possible for MSL when accommodating its larger landing footprint.

\section{Conclusions}

The Mars 2020 mission plans to continue the science exploration of the successful 2012 Mars Science Laboratory mission, while leveraging the same entry, descent, and landing architecture. However, the Mars 2020 mission also plans to improve upon the landing technologies pioneered by Mars Science Laboratory, such as improving landing accuracy as measured by landing footprint size. One way Mars 2020 will demonstrate 
improvements in landing technology is by deploying its parachute on a range trigger as opposed to the velocity trigger used on MSL. The simple change in triggering scheme leads to a vast decrease in landing ellipse size and more importantly shuffles the contributions of various models on trajectory dispersions. Capsule aerodynamic uncertainties that play a large role in ellipse sizes of ballistic, uncontrolled lifting bodies and velocity triggered vehicles no longer have a large role in the landed ellipse size. This has a consequence for improvements in future entry guidance schemes, since once range triggers are used with current Mars entry, descent, and landing settings, there is very little effect entry schemes can have on further reducing touchdown ellipse sizes, especially if the architecture includes uncontrolled descent phases, such as parachute descent. Instead, simulation results suggest that future improvements to ellipse sizes can be gained by focusing on guidance schemes that reduce atmospheric-related errors caused during drift on parachute or other terminal descent phase.

\section{References}

${ }^{1}$ Mustard, J., Adler, M., Allwood, A., Bass, D., Beaty, D., Bell III, J., Brinckerhoff, W., Carr, M., Des Marais, D., Drake, B., Edgett, K., Eigenbrode, J., Elkins-Tanton, L., Grant, J., Milkovich, S. M., Ming, D., Moore, C., Murchie, S., Onstott, T., Ruff, S., Sephton, M., Steele, A., and Treiman, A., "Report of the Mars 2020 Science Definition Team," Tech. rep., Mars Exploration Program Analysis Group (MEPAG), 2013.

${ }^{2}$ Steltzner, A., Kipp, D., Chen, A., Burkhart, D., Guernsey, C., Mendeck, G., Mitcheltree, R., Powell, R., Rivellini, T., Martin, M. S., and Way, D., "Mars Science Laboratory Entry, Descent, and Landing System," IEEEAC 1497, IEEE Aerospace Conference, Big Sky, MT, 2006.

${ }^{3}$ Prakash, R., Burkhard, P., Chen, A., Comeaux, K. A., Guernsey, C., Kipp, D., Lorenzoni, L., Mendeck, G., Powell, R., Rivellini, T., San Martin, M., Sell, S., Steltzner, A., and Way, D., "Mars Science Laboratory Entry, Descent, and Landing System Overview," IEEE AC 1531, IEEE Aerospace Conference, Big Sky, MT, 2008.

${ }^{4}$ Vasavada, A., Chen, A., Barnes, J., Burkhard, P., Canton, B., Dwyer-Cianciolo, A., Fergason, R., Hinson, D., Justh, H., Kass, D., Lewis, S., Mischna, M., Murphy, J., Rakfin, S., Tyler, D., and Withers, P., "Assessment of Environments for Mars Science Laboratory Entry, Descent, and Surface Operations," Space Science Review, Vol. 170, No. 1-4, 2012, pp. 793-835.

${ }^{5}$ Grover III, M., Cichy, B., and Desai, P., "Overview of Phoenix Entry, Descent, and Landing System Architecture," AIAA-2008-7218, AIAA Guidance, Navigation, and Control Conference, Honolulu, HI, 2008.

${ }^{6}$ Mendeck, G. and McGrew, L., "Entry Guidance Design and Postlfight Performance for 2011 Mars Science Laboratory Mission," Journal of Spacecraft and Rockets, Vol. 51, No. 4, 2014, pp. 1094-1105.

${ }^{7}$ Mendeck, G. and Carman, G., "Design for Mars Smart Landers Using the Entry Terminal Point Controller," AAS 2002-4502, AIAA Atmospheric Flight Mechanics Conference, Monterrey, CA, 2002.

${ }^{8}$ Way, D., "On the Use of a Range Trigger for the Mars Science Laboratory Entry, Descent, and Landing," IEEE AC 1142, IEEE Aerospace Conference, Big Sky, MT, 2011.

${ }^{9}$ Braun, R. D., Powell, R. W., Engelund, W. C., Gnoffo, P. A., Weilmuenster, K. J., and Mitcheltree, R. A., "Mars Pathfinder Six-Degree-of-Freedom Entry Analysis," Journal of Spacecraft and Rockets, Vol. 32, No. 6, 1995, pp. 993-1000.

${ }^{10}$ Desai, P. N., Schoenenberger, M., and Cheatwood, F. M., "Mars Exploration Rover Six-Degree-of-Freedom Entry Trajectory Analysis," Journal of Spacecraft and Rockets, Vol. 43, No. 5, Sept. 2006, pp. 1019-1025.

${ }^{11}$ Desai, P. N., Prince, J. L., Queen, E. M., Schoenenberger, M., Cruz, J. R., and Grover, M. R., "Entry, Descent, and Landing Performance of the Mars Phoenix Lander," Journal of Spacecraft and Rockets, Vol. 48, No. 5, 2011, pp. 798-808.

${ }^{12}$ Way, D. W., Davis, Jody, D., and Shidner, J. D., "Assessment of the Mars Science Laboratory Entry, Descent, and Landing Simulation," AAS 13-420, AAS/AIAA Space Flight Mechanics Conference, Kauai, HI, 2013.

${ }^{13}$ Chen, A., Vasavada, A., Cianciolo, A., Barnes, J., Tyler, D., Rafkin, S., Hinson, D., and Lewis, S., "Atmospheric Risk Assessment for the Mars Science Laboratory Entry, Descent, and Landing System," IEEEAC 1153, IEEE Aerospace Conference, Big Sky, MT, 2010.

${ }^{14}$ Striepe, S., Way, D., Dwyer, A. M., and Balaram, J., "Mars Science Laboratory Simulations for Entry, Descent, and Landing," Journal of Spacecraft and Rockets, Vol. 43, No. 2, March 2006, pp. 311-323.

${ }^{15}$ Martin-Mur, T., Kruizinga, G., and Wong, M., "Mars Science Laboratory Interplanetary Navigation Performance," AAS 13-232, AAS/AIAA Space Flight Mechanics Conference, Kauai, HI, 2013. 